\title{
Voronoi Diagrams with Respect to Criteria on Vision Information
}

\author{
Tetsuo Asano*, Naoki Katoh ${ }^{\dagger}$, Hisao TAmaki ${ }^{\ddagger}$ and Takeshi TokUyAma ${ }^{\S}$ \\ * School of Information Science, JAIST \\ 1-1 Asahidai, Nomi 923-1292, Japan \\ E-mail: t-asano@jaist.ac.jp \\ $\dagger$ Graduate School of Engineering, Kyoto University \\ Katsura, Nishikyo-ku, Kyoto 615-8540, Japan \\ E-mail: naoki@archi.kyoto-u.ac.jp \\ ¥Department of Computer Science, Meiji University \\ Higashimita, Tama-ku, Kawasaki 214-8571, Japan \\ E-mail: tamaki@cs.meiji.ac.jp \\ $\S$ Graduate School of Information Sciences, Tohoku University \\ Aramaki Aza-Aoba, Aoba, Sendai 980-8579, Japan \\ E-mail: tokuyama@dais.is.tohoku.ac.jp
}

Received July 9, 2007

Revised September 6, 2007

\begin{abstract}
Voronoi diagrams for a set of geometric objects is a partition of the plane (or space in higher dimensions) into disjoint regions each dominated by some given object under a predetermined criterion. In this paper we are interested in various measures associated with criteria on goodness of an input line segment with respect to each point in the plane as the "point of view." These measures basically show how well a segment or information displayed on the segment can be seen from the point. Mathematically, the measures are defined in terms of the shapes of the triangle determined by the point and the line segment. We study the combinatorial and algorithmic complexities of those Voronoi diagrams. We also study an associated optimization problem: find a point that maximizes the smallest measure value over the measures with respect to all the given line segments. We give sufficient conditions for an optimal point to lie on a Voronoi edge and present a heuristic optimization algorithm for those measures having this property.
\end{abstract}

Key words: algorithms, computational geometry, measure on triangles, optimization, triangle, visual angle, aspect ratio, minimal height, Voronoi diagram

\section{Introduction}

Given a set of points in the plane, we can partition the plane into regions in such a way that any point in a region associated with some given point is closer to the point than to any other point in the set. The resulting partition of the plane is called a Voronoi diagram for the point set. Replacing the relation "closer to" with some other criteria we could define a number of variations of the diagram (see e.g. $[4,11])$.

In this paper we propose a yet another abstraction of those Voronoi diagrams for a set of non-intersecting line segments possibly forming a polygon. We consider a measure associated with a criterion on how an input segment can be seen from a point in the plane as a point of view. There are several possible criteria. Naturally, the distances from the segments and the lengths of segments are important factors. If two segments are of the same length, one may conclude that the nearer one should be seen better; however, it is not always the case, since if we see a blackboard from 
the leftmost seat in the first row of a classroom, we have difficulty to read letters written on the blackboard. Also, liquid-crystal display of a laptop computer can be only seen from points in a limited region. Basically, the visual quality of information given on a line segment $s$ seen from a point $p$ depends on the shape of triangle $\triangle(p, s)$ defined by $s$ and $p$ such that $\triangle(p, s)$ has $s$ as an edge and $p$ as its opposite vertex. We consider $\mu(p, s)$ to measure the quality of the visual information on $s$ obtained at $p$. Each measure $\mu$ may be a goodness measure, with larger values meaning better quality, or a badness measure, with larger values meaning poorer quality. For example, in usual situations, the distance between $p$ and $s$ is a badness measure, while the visual angle of $s$ at $p$, defined to be the internal angle of vertex $p$ of the triangle $\triangle(p, s)$, is a goodness measure. Note, however, that the same measure may serve as both goodness and badness measures in different situations.

Suppose we are given a set $S=\left\{s_{i}\right\}$ of line segments and a measure $\mu$. For each point $p$ in the plane, we are concerned with the segment $s_{i}$ for which the value $\mu\left(p, s_{i}\right)$ is the worst of $\mu\left(p, s_{j}\right)$ over all $s_{j}$, since that segment may present a bottleneck in processing the visual information from all the segments. If $\mu$ is a goodness measure, this naturally gives rise to the Voronoi diagram for which $\mu(p, s)$ is regarded as the distance between $p$ and $s$ : the Voronoi region of $s_{i}$ consists of those points $p$ for which $\mu\left(p, s_{i}\right)<\mu\left(p, s_{j}\right)$ for each $s_{j} \in S$ distinct from $s_{i}$. If $\mu$ is a badness measure, for the same reason, we are instead interested in the farthestpoint Voronoi diagram, for which the direction of the inequality is flipped. In the following, we call Voronoi diagrams of the first type minimum-measure Voronoi diagrams and those of the second type maximum-measure Voronoi diagrams. When it is clear from the context whether measure $\mu$ is a goodness measure or a badness measure, we omit the minimum-measure/maximum-measure distinction and simply say the Voronoi diagram associated with $\mu$.

The Voronoi diagram associated with a goodness or badness measure is a rich source of information regarding questions with respect to the measure and the point set. In particular, it can be used to solve a certain type of optimization problems. For a goodness measure $\mu$, we are interested in the max-min optimization: find a point $p$ that maximizes the minimum of $\mu\left(p, s_{i}\right)$ over all $s_{i} \in S$. In the visual information setting, we look for a point from which we best see the worst-seen object. For a badness measure, we are interested in the min-max optimization defined similarly. We give some sufficient condition on the measure in order for such an optimal point to always lie on some Voronoi edge.

With a different criterion we can define a similar but different Voronoi diagram. Voronoi edges are characterized in a different manner. The purpose of this paper is to find combinatorial and structural properties common to all those Voronoi diagrams associated with measures $\mu$ defined for a pair of point and line segment. We describe basic properties to be satisfied by the measures to possess those common properties. It is important for practical use. There may be a number of problems falling into the class which can be solved using our framework of Voronoi diagrams. Although it is impossible to enumerate all possible optimization criteria, it is pos- 
sible to investigate basic conditions to be satisfied by those criteria in order to have their corresponding Voronoi diagrams bear the same combinatorial properties.

An original motivation of this Voronoi diagram comes from applications to mesh improvement and robotics. Mesh generation/improvement [5, 6, 10, 12, 13] is an important process for many purposes including Finite Element Method. In a simple setting, a given simple polygon is partitioned into many small triangles after inserting an appropriate number of points in its interior as vertices of triangular meshes. Several different criteria have been considered to evaluate the quality of such a triangular mesh. One of them is to maximize the smallest internal angle (or to minimize the largest internal angle). Since polygon vertices are fixed, the only way to improve the quality of triangular mesh is either to move internal points or to insert new internal points (or even delete existing internal points). In robotics, we are interested in locating a robot in the amidst of many polygonal obstacles by computing its relative position to the most outstanding polygon or line segment in a criterion on visual information.

The Voronoi diagram is also given as the lower (or upper) envelope of terrains, where a terrain for a line segment $s_{i}$ is defined using $\mu\left(p, s_{i}\right)$ as height at the point $p$. A general theory on terrains by Halperin and Sharir [7] yields an upper bound $O\left(n^{2+\varepsilon}\right)$ on the complexity of the lower envelope of those terrains that is Voronoi diagram, where $\varepsilon$ is an arbitrarily small positive constant. In other words, the Voronoi diagram has $O\left(n^{2+\varepsilon}\right)$ Voronoi edges, and vertices. Despite the high complexity in the worst case, actual complexity seems to be low by our experiments for a number of polygons.

This paper is organized as follows. In Section 2 we define a Voronoi diagram associated with an abstract measure $\mu$ on a triangle. In Section 3 a list of possible measures is given together with algebraic expressions for those measures. In Section 4 , we study the complexity of the Voronoi diagrams under those measures. In Section 5, we study the associated optimization problems. Finally, Section 6 gives some concluding remarks together with some open problems and future works.

\section{Voronoi diagrams for various criteria on triangles}

In the following definitions, we deal with minimum-measure Voronoi diagrams. The definitions for maximum measure Voronoi diagrams are obtained by flipping the directions of the inequalities.

Suppose we are given a set $S=\left\{s_{i}\right\}$ of line segments on the plane and a measure $\mu$ on $R \times S$. A point $p$ is said to be dominated by a line segment $s_{i} \in S$ if

$$
\mu\left(p, s_{i}\right)=\min \left\{\mu\left(p, s_{j}\right) \mid s_{j} \in S\right\}
$$

For each $s_{i} \in S$, the Voronoi region $V\left(s_{i}\right)$ is defined to be the set of points singly dominated by $s_{i}$, i.e.,

$$
V\left(s_{i}\right)=\left\{p \in \mathbb{R}^{2} \mid \mu\left(p, s_{i}\right)<\mu\left(p, s_{j}\right) \text { for any } j \neq i\right\} .
$$


Voronoi edges are defined by curves which are dominated by exactly two elements of $S$. Formally, the Voronoi edge $E\left(s_{i}, s_{j}\right)$ is defined by

$$
E\left(s_{i}, s_{j}\right)=\left\{p \in \mathbb{R}^{2} \mid \mu\left(p, s_{i}\right)=\mu\left(p, s_{j}\right)<\mu\left(p, s_{k}\right) \text { for any } k \neq i, j\right\} .
$$

Two Voronoi edges may meet at one point, that is a Voronoi vertex. It is defined by

$$
v\left(s_{i}, s_{j}, s_{k}\right)=\left\{p \in \mathbb{R}^{2} \mid \mu\left(p, s_{i}\right)=\mu\left(p, s_{j}\right)=\mu\left(p, s_{k}\right) \leq \mu\left(p, s_{l}\right) \text { for any } l \neq i, j, k\right\},
$$

which is a set of points dominated by three or more elements of $S$.

As in the usual definition of generalized Voronoi diagrams, we require that the Voronoi regions form a tessellation of the entire plane: $V\left(s_{i}\right) \cap V\left(S_{j}\right)=\emptyset$ for $s_{i} \neq s_{j}$ and $\bigcup_{s_{i} \in S} \overline{V\left(s_{i}\right)}=R^{2}$, where $\overline{V\left(s_{i}\right)}$ denotes the closure of the Voronoi region $V\left(s_{i}\right)$.

For some measures, this requirement is violated by some degenerate configurations of line segments. For example, consider a measure $\mu\left(p, s_{i}\right)$ defined to be the distance from point $p$ to the line including line segment $s_{i}$. If two line segments $s_{i}$ and $s_{j}$ lie on a line $l, \mu\left(p, s_{i}\right)=\mu\left(p, s_{j}\right)$ for any point $p$. Thus, if the set $S$ contains such co-linear pair of line segments, the resulting Voronoi regions may not form a tessellation. We exclude those degenerate configurations from our considerations. In practice, we may avoid them by small perturbations.

For yet some other measures, however, the above tessellation requirement is more severely violated, in the sense that small perturbations may not resolve the problem. This may happen when, for example, the measure with respect to a line segment takes a constant value for all points in some region. We will later see an example of well-motivated measures in this category. When those measures are used, we need some extra care outside of the framework presented in this paper.

For the sake of the analysis of the complexity of the Voronoi diagrams and their constructions, we will impose the standard assumption that the measure is described by a constant number of algebraic functions. We give more details in Section 4.

For our application on visual information, it may make more sense to include visibility considerations in the definition of the Voronoi diagram: a point $p$ belongs to the Voronoi region of $s_{i}$ if $s_{i}$ is entirely visible from $p$ (without being hidden by other line segments) and $\mu\left(p, s_{i}\right)<\mu\left(p, s_{j}\right)$ for every $s_{j} \neq s_{i}$ that is entirely visible from $p$. This modification would make the Voronoi diagrams more difficult to analyze: we need at least to deals with the possibilitye of some points not dominated by any segment. In this paper, we are mainly concerned with the basic Voronoi diagrams without visibility considerations. 


\section{A list of possible measures}

We say that measure $\mu$ is symmetric if for each triangle $p q r$ we have $\mu(p, q r)=$ $\mu(q, r p)=\mu(r, p q)$. For any measure $\mu$, there are symmetric versions $\mu^{\max }$ and $\mu^{\text {min }}$, defined by

$$
\mu^{\max }(p, q r)=\max \{\mu(p, q r), \mu(q, r p), \mu(r, p q)\},
$$

and

$$
\mu^{\min }(p, q r)=\min \{\mu(p, q r), \mu(q, r p), \mu(r, p q)\}
$$

We first list a few asymmetric measures. All of them can be used as both goodness and badness measures depending on the situations.

visual angle Define $\mu_{1}\left(p, s_{i}\right)=\theta_{p}\left(s_{i}\right)$ which is defined to be the visual angle of $s_{i}$ from $p$.

height Define $\mu_{2}\left(p, s_{i}\right)$ as the distance from $p$ to the line containing $s_{i}$.

aspect-ratio Define $\mu_{3}\left(p, s_{i}\right)=\mu_{2}\left(p, s_{i}\right) /\left\|s_{i}\right\|$, where $\left\|s_{i}\right\|$ denotes the length of segment $s_{i}$.

These asymmetric measures are natural for visual information applications, while their symmetric versions are more appropriate for mesh optimization applications: $\mu_{1}^{\min }, \mu_{2}^{\min }$, and $\mu_{3}^{\min }$ are all goodness measures and $\mu_{1}^{\max }, \mu_{2}^{\max }$, and $\mu_{3}^{\max }$ are all badness measures. The following are inherently symmetric badness measures.

circumcircle Define $\mu_{4}\left(p, s_{i}\right)$ to be the radius of the circumcircle of a triangle defined by $\left(p, s_{i}\right)$.

eccentricity Define $\mu_{5}\left(p, s_{i}\right)$ as follows: it is 0 if the center of the circumcircle of a triangle $\triangle\left(p, s_{i}\right)$ lies in the interior of the triangle. Otherwise, it is the distance from the center to the closest edge of the triangle.

Fig. 1 illustrates some of the listed measures: $\mu_{1}, \mu_{2}^{\min }, \mu_{3}^{\min }, \mu_{4}$, and $\mu_{5}$.

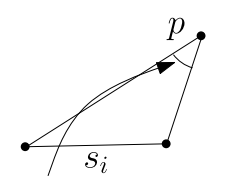

(a) $\mu_{1}\left(p, s_{i}\right)$

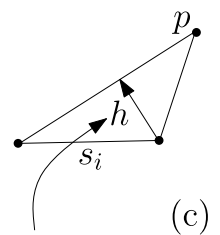

(c) $\mu_{3}^{\min }\left(p, s_{i}\right)=h / L$

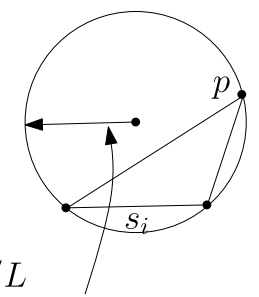

(d) $\mu_{4}\left(p, s_{i}\right)=r$

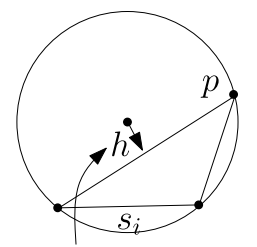

(e) $\mu_{5}\left(p, s_{i}\right)=h$

(b) $\mu_{2}^{\min }\left(p, s_{i}\right)=h$

Fig. 1. A list of measures on triangles.

Note that the last measure eccentricity may violate the tessellation requirement discussed in Section 2, due to the constant value 0 for $\left(p, s_{i}\right)$ forming an acute triangle. 


\subsection{Corresponding Voronoi diagrams}

Fig. 2 shows Voronoi diagrams for some of the measures listed above. The last one shown in (f) takes visibility constraints into account, as described in Section 2. The authors implemented a C program using LEDA [8] to obtain those figures. For simplicity those diagrams are drawn pixel by pixel instead of drawing Voronoi edges following equations of corresponding planar curves. In the figures line segments (polygon edges) have different colors (darkness). Regions associated with a line segment are painted by the colors (darkness) associated with it.

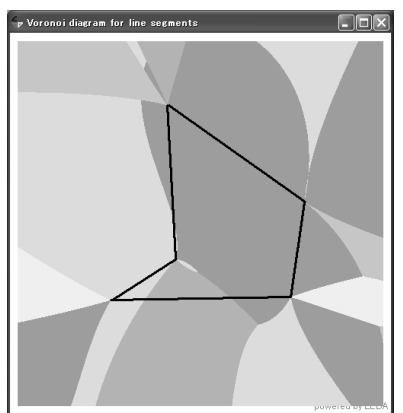

(a)

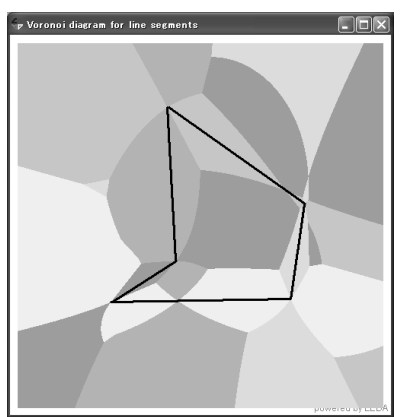

(d)

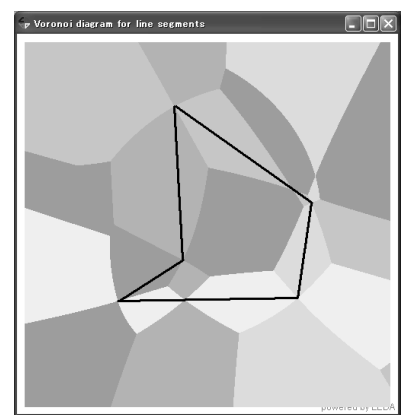

(b)

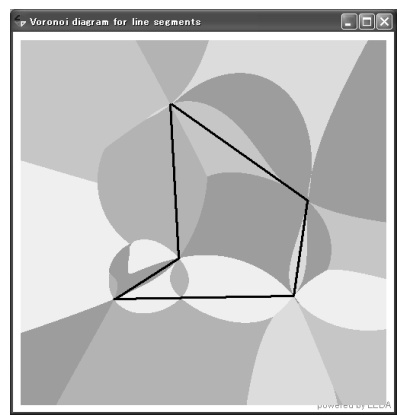

(e)

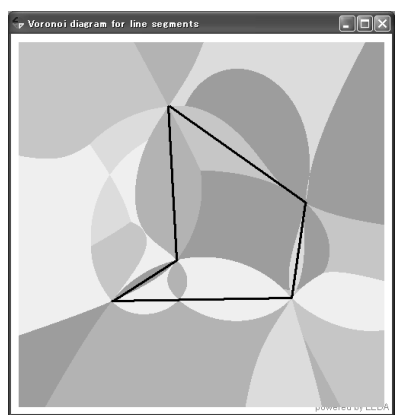

(c)

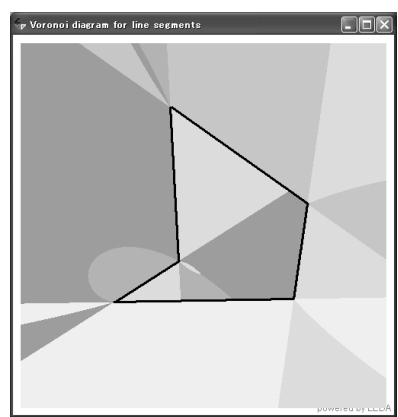

(f)

Fig. 2. Voronoi diagrams for various measures: (a) visual angle $\left(\mu_{1}\right)$ as a goodness measure, (b) minimum height $\left(\mu_{2}^{\min }\right)$, (c) minimum aspect ratio $\left(\mu_{3}^{\min }\right)$, (d) circumcircle $\left(\mu_{4}\right),(\mathrm{e})$ eccentricity $\left(\mu_{5}\right)$, and (f) visual angle as a goodness measure, with visibility constraint.

Although eccentricity may violate the tessellation requirement in general, the line segment configuration in the example does not cause this problem.

Fig. 3 shows another pair of examples. The measure used for the diagrams is the distance of the point to the line segment. The visibility condition is considered in the right figure (b), but not in the left one (a). This is known as a Voronoi diagram for a set of line segments. Although the line segments appear to form a polygon, they are slightly perturbed so that no endpoints of two line segments coincide. If two line segments have a common endpoint, there will be a region of points equidistant to those two line segments, violating the tessellation requirement. 


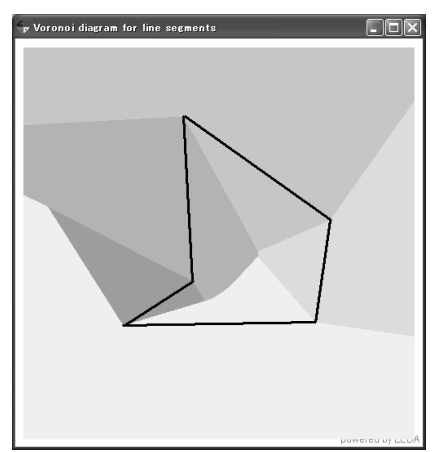

(a)

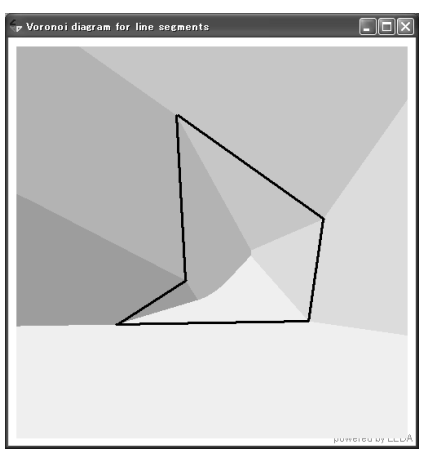

(b)

Fig. 3. Voronoi diagrams for the usual distance measure. Visibility constraint is taken into consideration in the right diagram (b) but not in the left one (a).

\subsection{Algebraic expressions}

Below we list explicit algebraic expressions for the measures listed earlier. They are needed in the algorithms for construction, and for the analysis of the complexity of associated Voronoi diagrams.

visual angle Although the visual angle itself cannot be expressed as an algebraic function, its cosine value $\mu_{1}^{\prime}\left(p, s_{i}\right)=\cos \theta_{p}\left(s_{i}\right)$ can, which is sufficient for our purpose of constructing the Voronoi diagram and doing optimization. Exactly, we have

$$
\cos \theta_{p}\left(s_{i}\right)=\frac{\|p a\|^{2}+\|p b\|^{2}-\|a b\|^{2}}{2\|p a\| \cdot\|p b\|},
$$

where $a$ and $b$ are two endpoints of $s_{i}$ and $\|p q\|$ denotes the length of the segment $\overline{p q}$. Assuming $p=(x, y), a=\left(a_{x}, a_{y}\right)$, and $b=\left(b_{x}, b_{y}\right)$, we have

$$
\begin{aligned}
& \cos \theta_{p}\left(s_{i}\right) \\
& =\frac{\left(x-a_{x}\right)^{2}+\left(y-a_{y}\right)^{2}+\left(x-b_{x}\right)^{2}+\left(y-b_{y}\right)^{2}-\left(a_{x}-b_{x}\right)^{2}-\left(a_{y}-b_{y}\right)^{2}}{2 \sqrt{\left(x-a_{x}\right)^{2}+\left(y-a_{y}\right)^{2}} \sqrt{\left(x-b_{x}\right)^{2}+\left(y-b_{y}\right)^{2}}} .
\end{aligned}
$$

height Letting $A$ be the area of the triangle $\triangle\left(p, s_{i}\right)$ and $L$ be the length of $s_{i}$, the height $\mu_{2}\left(p, s_{i}\right)$ of $p$ with respect to $s_{i}$ is given by:

$$
\begin{aligned}
\mu_{2}\left(p, s_{i}\right) & =2 A / L \\
& =\frac{\left|\left(a_{y}-b_{y}\right) x+\left(b_{x}-a_{x}\right) y+a_{x} b_{y}-b_{x} a_{y}\right|}{\sqrt{\left(a_{x}-b_{x}\right)^{2}+\left(a_{y}-b_{y}\right)^{2}}} .
\end{aligned}
$$

aspect-ratio With the same notation as above, we have:

$$
\begin{aligned}
\mu_{3}\left(p, s_{i}\right) & =\mu_{2}\left(p, s_{i}\right) / L \\
& =2 A / L^{2} \\
& =\frac{\left|\left(a_{y}-b_{y}\right) x+\left(b_{x}-a_{x}\right) y+a_{x} b_{y}-b_{x} a_{y}\right|}{\left(a_{x}-b_{x}\right)^{2}+\left(a_{y}-b_{y}\right)^{2}} .
\end{aligned}
$$


circumcircle It is known that the radius of a triangle $p a b$ with area $A$ is given by

$$
\begin{aligned}
& \mu_{4}\left(p, s_{i}\right) \\
& =\frac{\|p a\| \cdot\|p b\| \cdot\|a b\|}{4 A} \\
& =\frac{\sqrt{\left(x-a_{x}\right)^{2}+\left(y-a_{y}\right)^{2}} \sqrt{\left(x-b_{x}\right)^{2}+\left(y-b_{y}\right)^{2}} \sqrt{\left(a_{x}-b_{x}\right)^{2}+\left(a_{y}-b_{y}\right)^{2}}}{2\left|\left(a_{y}-b_{y}\right) x+\left(b_{x}-a_{x}\right) y+a_{x} b_{y}-b_{x} a_{y}\right|} .
\end{aligned}
$$

eccentricity Let $r$ be the radius of the circumcircle of a triangle $\triangle p a b$. If the center of the circumcircle lies in the interior of the triangle, $\mu_{5}\left(p, s_{i}\right)=0$. Otherwise, $\mu_{5}\left(p, s_{i}\right)$ is the smallest of the distances from the center to the segment $\overline{p a}, \overline{p b}$, and $s_{i}$. Thus, $\mu_{5}\left(p, s_{i}\right)$ is expressed as a combination of six algebraic functions, three for the distance from the center to the lines containing the sides of the triangle and three for the distance from the center to the vertices of the triangle. We omit the exact expression here.

\section{Complexity of Voronoi diagrams}

The purpose of this section is to study the combinatorial and algorithmic complexities of our Voronoi diagrams. We first note that the Voronoi region $V\left(s_{i}\right)$ for each $s_{i}$ may not be connected. For example, let us take the visual angle as the measure and consider minimum-measure Voronoi diagrams. Fig. 4 shows an example with three line segments in which Voronoi regions are indeed disconnected.

Fig. 5 illustrates a configuration for which the complexity of the diagram is quadratic.

To get an upper bound on the complexity of our Voronoi diagrams, we apply the result of Halperin and Sharir [7] on the complexity of the lower envelope of terrains defined by algebraic functions.

Let $f_{i}, 1 \leq i \leq n$, be bivariate algebraic function of a constant degree, possibly partially defined. Assume that, when $f_{i}$ is partially defined, the domain of the function is bounded by an algebraic curve of a constant degree. Then their result says that the complexity of the lower envelope of the terrains defined by $z=f_{i}(x, y)$, $1 \leq i \leq n$, is $O\left(n^{2+\varepsilon}\right)$ for any positive constant $\varepsilon$. It is also known that the envelope can be computed in $O\left(n^{2+\varepsilon}\right)$ time [1] using a divide and conquer strategy.

Our minimum-measure Voronoi diagram is obtained by projecting the lower envelope of the terrains defined by $z=g_{i}(x, y)=\mu\left((x, y), s_{i}\right), s_{i} \in S$, to the $x y$-plane. If $\mu$ is defined by a single algebraic function, then we can immediately apply the above results. For more general cases where $\mu$ is defined by a combination of a constant number of algebraic functions, we need to guarantee that the domain of each constituent function is bounded by an algebraic curve of a constant degree. To do so, it suffices to assume that $\mu\left(p, s_{i}\right)$ for fixed $s_{i}$ is continuous except on $s_{i}$. 


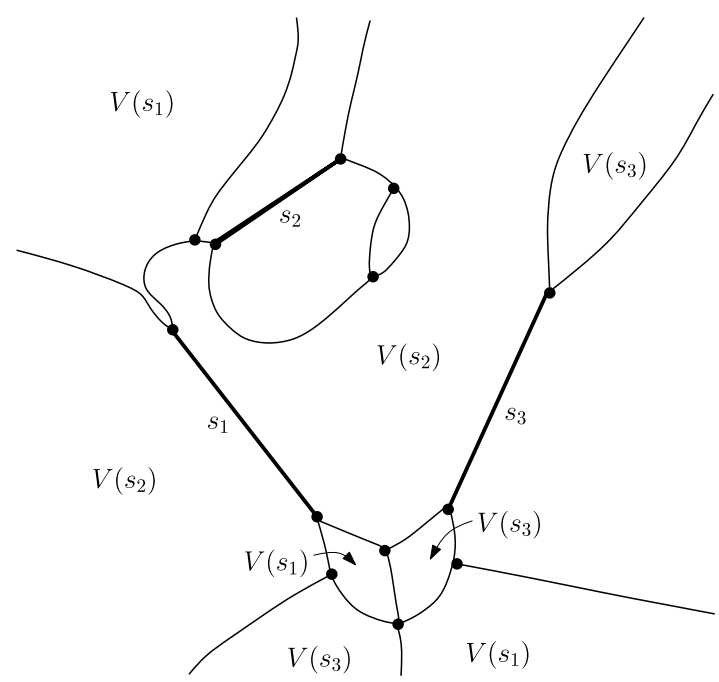

Fig. 4. A Voronoi diagram for a set $S$ of three line segments (shown by bold lines) under the measure of max-min visual angle. A point belongs to a Voronoi region dominated by a line segment giving the smallest visual angle.

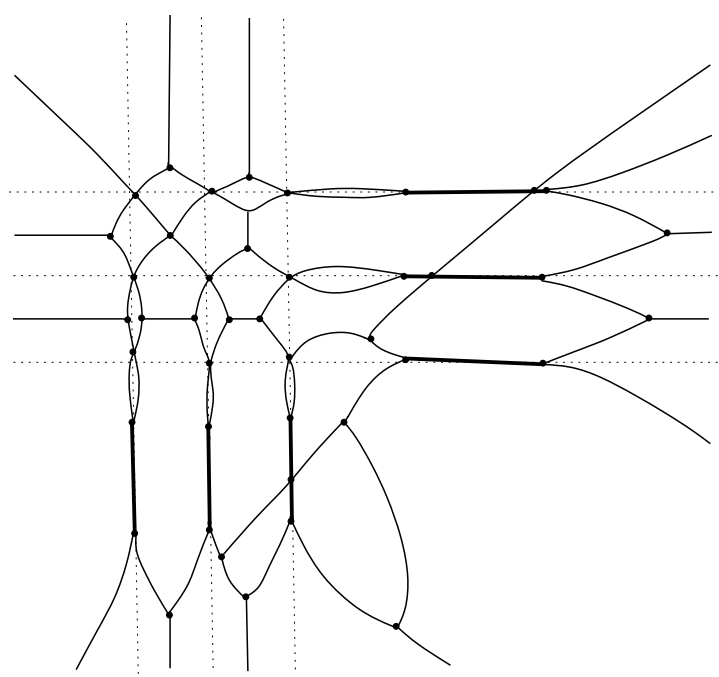

Fig. 5. A visual angle Voronoi diagram for a set $S$ of horizontal and vertical line segments (shown by bold lines) having quadratic number of Voronoi edges and vertices. Dotted lines in the figure are extensions of given line segments. Segment endpoints are shown by dots in the figure. 
TheOREm 1. Let $\mu$ be a measure such that, for each fixed line segment $s$, $\mu(p, s)$ is a piecewise algebraic function on $p$ of a constant degree consisting of a constant number of pieces and is continuous except on s. Assume further that for some set $S$ of $n$ line segments, the pair $(\mu, S)$ satisfies the tessellation requirement. Then, the Voronoi diagram of $S$ under $\mu$ consists of $O\left(n^{2+\varepsilon}\right)$ cells, edges, and vertices, where $\varepsilon$ is an arbitrarily small positive constant, and it can be computed in $O\left(n^{2+\varepsilon}\right)$ time.

It is straightforward to verify that all the measures listed above (except for visual angle $\mu_{1}$ which should be replaced by the cosine version $\mu_{1}^{\prime}$ ) satisfy the assumption of this theorem.

\section{Optimization}

Given a goodness measure $\mu(p, s)$ and a set of line segments $S=\left\{s_{i}\right\}$, our Voronoi diagram gives us a means for computing $\min \left\{\mu\left(p, s_{i}\right) \mid s_{i} \in S\right\}$ for each $p$. As discussed in the introduction, we are interested in finding a point $p$ that maximizes this function. For badness measures, we are interested in minimizing the $\max \left\{\mu\left(p, s_{i}\right) \mid s_{i} \in S\right\}$. In the following, we deal with goodness measures and associated max-min optimization problems. Badness measures and associated min-max optimization problems can be similarly dealt with.

We first give simple sufficient conditions for an optimal point to lie on a Voronoi edge. Given a measure $\mu$ and a fixed line segment $s$, a point $p^{*}$ is a peak of $\mu$ with respect to $s$, if it gives a local optimum of $\mu(p, s)$ as a function on $p$ : for any $p$ in the neighborhood of $p^{*}, \mu\left(p^{*}, s\right) \geq \mu(p, s)$. It is clear that if a measure does not have any peak then optimal points of the max-min optimization problem must lie on Voronoi edges.

We say that measure $\mu$ satisfies the single peak value property, if there is some universal constant $c$ such that for every line segment $s$ we have:

1. $\mu(p, s) \leq c$ for every point $p$, and

2. every local optimum of $\mu(p, s)$ takes the value $c$.

THEOREM 2. Let $\mu$ be a goodness measure satisfying the single peak value property and let $S$ be some set of line segments. Then, each optimal point of the associated max-min optimization problem lies on a Voronoi edge of the Voronoi diagram for $S$ under $\mu$.

Proof. Let $p^{*}$ be a local optimum point for some $s_{i} \in S$. From the definition of the single peak value property, we have $\mu\left(p^{*}, s_{j}\right) \leq \mu\left(p^{*}, s_{i}\right)$ for every $s_{j} \in S$, which prohibits $p^{*}$ from lying in the Voronoi region of $s_{i}$. Therefore, each optimal point must lie on some Voronoi edge.

Let us consider whether the concrete measures listed earlier satisfy the above sufficient conditions. The visual angle measure $\mu_{1}$, together with its symmetric versions $\mu_{1}^{\min }$ and $\mu_{1}^{\max }$, satisfies the single peak value property as both goodness and badness measures. The aspect ratio $\mu_{3}$ as a badness measure, together with its 
symmetric version $\mu_{3}^{\min }$, satisfies the single peak value property. The height $\mu_{2}$ as a goodness measure, together with its symmetric version $\mu_{2}^{\min }$ does not have peaks. However, the radius of circumcircle $\mu_{4}$ as a badness measure does have peaks (local minima) but does not satisfy the single peak value property. The peak value for $s_{i}$ is just the half of the length of the line segment since the circle with $s_{i}$ as its diameter is the smallest circle passing through the two endpoints of $s_{i}$. Thus, two line segments having different lengths have different peak values. Thus, $\mu_{4}$ does not satisfy the single peak value property. In fact, in the Voronoi diagram associated with the measure shown in Fig. 6 an optimal point is not located on Voronoi edges.

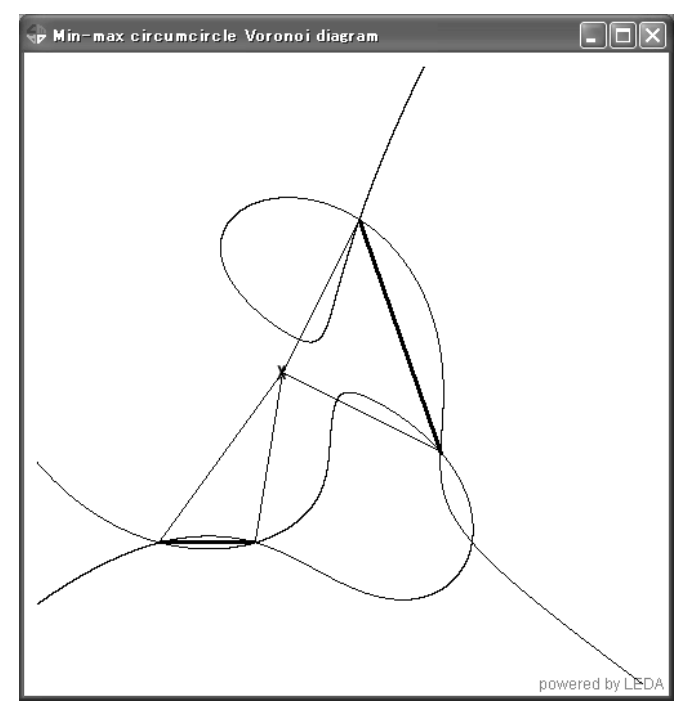

Fig. 6. A Voronoi diagram associated with the circumcircle radius as a badness measure for two line segments (drawn by bold lines). An optimal point that minimizes the radius of the maximum circumcircles is depicted by a cross and connected with four endpoints of the two line segments. The optimal point does not lie on a Voronoi edge.

For those measures satisfying the condition of the theorem, a natural $O\left(n^{2+\varepsilon}\right)$ time algorithm to solve the max-min optimization problem is to examine all Voronoi edges and compute the optimal point on each of them. Unfortunately, the time bound is rather large and indeed there is an algorithm with smaller running time. Our optimization problem can be formulated as an LP-type problem [9] and can be solved in almost linear time. Practical comparisons of these algorithms, however, have yet to be done through experimental methods.

In the rest of this section, we seek a heuristic algorithm that makes use of the Voronoi diagrams and their property that the optimal points are located on Voronoi edges. 


\section{Level region}

Given a goodness measure $\mu$, we define a level region by

$$
R_{>t}\left(s_{i}\right)=\left\{p \in \mathbb{R}^{2} \mid \mu\left(p, s_{i}\right)>t\right\} .
$$

That is, it consists of all points at which the measure is greater than some given value $t . R_{\geq t}\left(s_{i}\right)$ is similarly defined. Fig. 7 shows level regions for some measures listed earlier (although there is another region symmetric with respect to a line segment, only one of them is shown). For the measure $\mu_{1}$ on max-min visual angle, the level region $R_{\geq \theta}\left(s_{i}\right)$ is the interior of a circle on which the circular angle is exactly $\theta$, as shown in Fig. 7 (a). For the measure $\mu_{2}$ on max-min height, $R_{\geq h}\left(s_{i}\right)$ above the line segment $s_{i}$ is characterized by two lines each passing through an endpoint of the line segment and the line parallel to $s_{i}$ separated by $h$ from $s_{i}$. Thus, the region is an infinite region bounded by two rays and one line segment (which may be degenerated to a point). The measure $\mu_{3}$ on max-min aspect ratio has the level region $R_{\geq \alpha}\left(s_{i}\right)$ bounded by two circular arcs and one line segment parallel to $s_{i}$. The gap between two parallel lines is $\alpha \times\left\|s_{i}\right\|$ where $\left\|s_{i}\right\|$ is the length of $s_{i}$. The two circles determining the circular arcs have their center on lines perpendicular to $s_{i}$ and passing through the two endpoints of $s_{i}$. The level region for the measure on min-max circumcircle is not convex. It is bounded by two circular arcs of the same radius and both passing through the two endpoints of $s_{i}$. As is easily seen, whenever a point $p$ lies on the boundary, the circumcircle of the triangle $\triangle\left(p, s_{i}\right)$ is given by the circle shown in the figure.

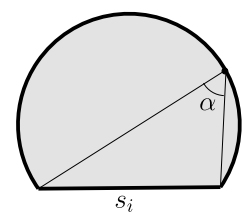

(a)

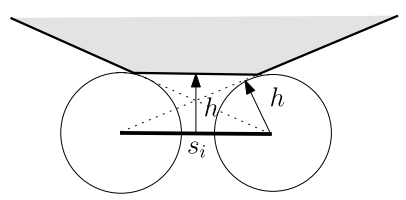

(b)

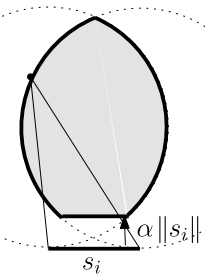

(c)

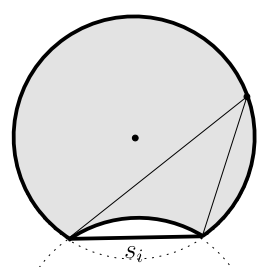

(d)

Fig. 7. Level regions $R_{\geq t}\left(s_{i}\right)$ for four different measures: (a) visual angle as a goodness measure, (b) minimum height, (c) minimum aspect-ratio, and (d) circumcircle as a badness measure.

For a line segment $s_{i}$ and a real value $t>0$, the level region $R_{\geq t}\left(s_{i}\right)$ appears in both sides of the line segment. In Fig. 7 we only illustrate one of the two regions since they are symmetric. Every boundary curve of the level region is described by a polynomial equation of constant degrees in $x$ and $y$.

\section{Heuristic algorithm}

The following is a heuristic algorithm for our optimization problem, guided by a Voronoi diagram associated with a given measure. For simplicity, we assume that the set of line segments form a convex or star-shaped polygon, but the algorithm can be adapted to more general cases. 


\section{PIXEL METHOD.}

(1) Given a convex polygon or a star-shaped polygon $P$, first distribute $k$ points $q_{1}, q_{2}, \ldots, q_{k}$ in its interior, where $k$ is some small constant.

(2) Compute the measures at those points.

(3) Take the best value $t$ among them.

(4) For each polygon edge $s_{i}$, compute the level region $R_{>t}\left(s_{i}\right)$.

(5) Take their intersection $R$ by plane sweep (typically in $O(n \log n)$ time).

(6) Enumerate all the Voronoi edges intersecting $R$. Note that if two level regions for $s_{i}$ and $s_{j}$ intersect on the boundary of $R$, then it means that the Voronoi edge $E\left(s_{i}, s_{j}\right)$ intersects the region $R$. In this way we can enumerate all the relevant Voronoi edges.

(7) For each such Voronoi edge, find its peak within $R$. Then, take the best value $t$ among them.

(8) If the region $R$ is small enough, stop the algorithm with the peak value $t$ found. Otherwise, go back to step (4).

Fig. 8 show how the feasible regions are computed for sets of points. In the figure, Voronoi diagrams for the measure on max-min aspect-ratio are superimposed with level regions for polygon edges. The boundaries of those level regions are depicted by bold lines. It is seen that Voronoi edges appear at vertices of the boundaries. It is also seen that given $k$ points in the interior of a polygon at least one point lies on the boundary and no point lies in its interior.
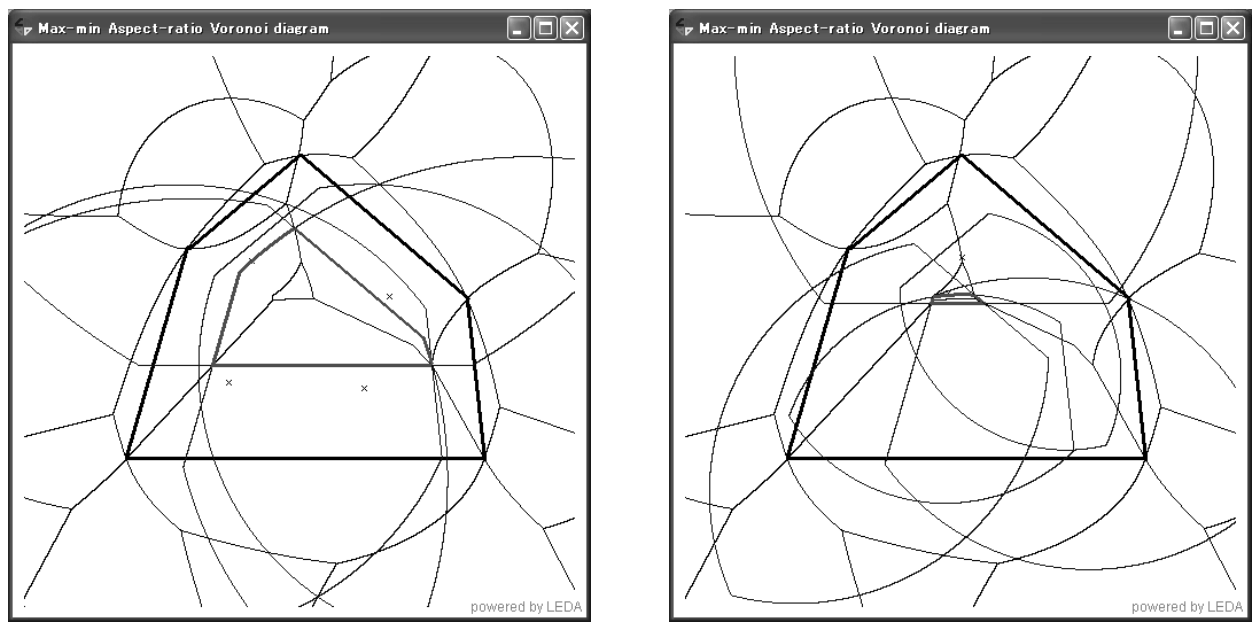

Fig. 8. Two sets of points (marked by crosses) distributed over a polygon and their associated feasible regions computed by the heuristic algorithm. 
Given $k$ points in the interior of a given polygon, we can compute the values $e\left(p_{1}\right), \ldots, e\left(p_{k}\right)$ in $O(k n)$ time. Then, compute intersection of $n$ level regions for the largest value among $e\left(p_{1}\right), \ldots, e\left(p_{k}\right)$.

The total time complexity depends on how many iterations we need as well as on the time complexity of the plane sweep, which in turn depends on the complexity of the level curves. There is no analysis on the running time.

Fig. 9 show a Voronoi diagram for the measure of max-min aspect ratio with colors and without colors.
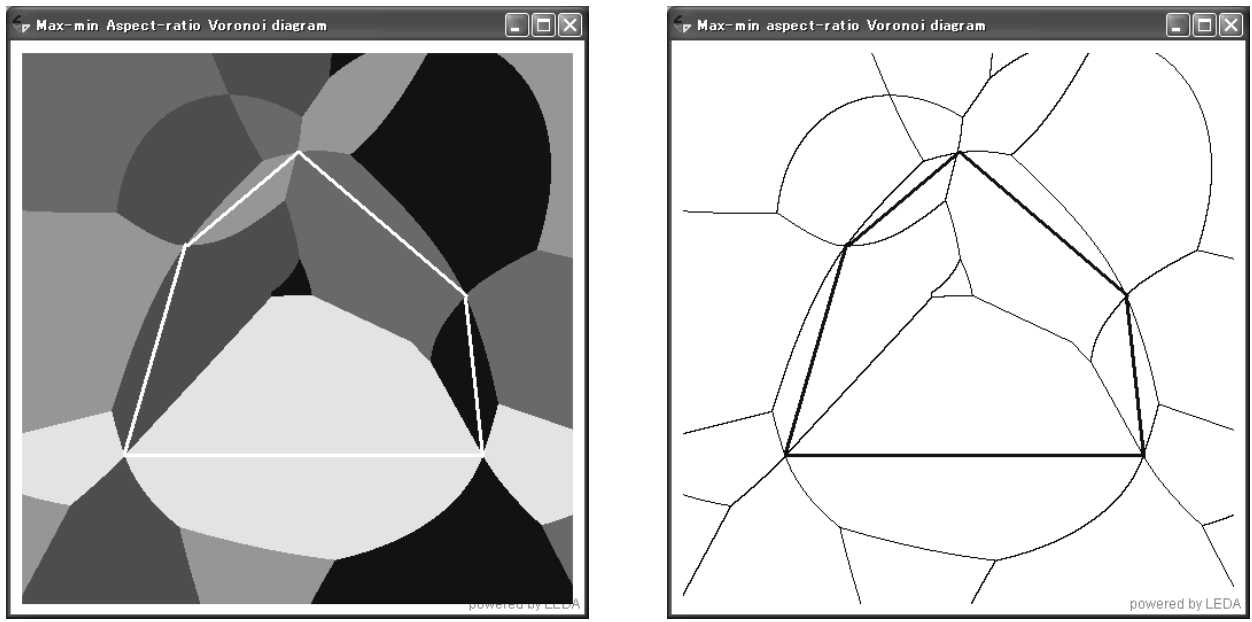

Fig. 9. Voronoi diagram for the measure of max-min aspect ratio with colors and without colors.

\section{Finding a peak on a Voronoi edge}

Both in the exact $O\left(n^{2+\varepsilon}\right)$ time algorithm and in the heuristic algorithm, we need to compute an optimal point on a single edge as a primitive operation. This is purely a matter of algebraic calculations. We illustrate this process with an example.

Take the minimum aspect ratio $\mu_{3}^{\min }$ as our measure $\mu$. We have:

$$
\begin{aligned}
& \mu\left(p, s_{i}\right) \\
& = \begin{cases}\frac{\left|\left(a_{y}-b_{y}\right) x+\left(b_{x}-a_{x}\right) y+a_{x} b_{y}-a_{y} b_{x}\right|}{\left(x-a_{x}\right)^{2}+\left(y-a_{y}\right)^{2}}, & \text { if } p a \text { is the longest side of } \triangle\left(p, s_{i}\right), \\
\frac{\left|\left(a_{y}-b_{y}\right) x+\left(b_{x}-a_{x}\right) y+a_{x} b_{y}-a_{y} b_{x}\right|}{\left(x-b_{x}\right)^{2}+\left(y-b_{y}\right)^{2}}, & \text { if } p b \text { is the longest side of } \triangle\left(p, s_{i}\right), \\
\frac{\left|\left(a_{y}-b_{y}\right) x+\left(b_{x}-a_{x}\right) y+a_{x} b_{y}-a_{y} b_{x}\right|}{\left(b_{x}-a_{x}\right)^{2}+\left(b_{y}-a_{y}\right)^{2}}, & \text { if } a b \text { is the longest side of } \triangle\left(p, s_{i}\right),\end{cases}
\end{aligned}
$$

where $p=(x, y)$ and $\left(a_{x}, a_{y}\right)$ and $\left(b_{x}, b_{y}\right)$ are the starting and ending points of $s_{i}$. We also have three different expressions for $\mu\left(p, s_{j}\right)$. Thus, there are 9 different 
forms of equations for $\mu\left(p, s_{i}\right)=\mu\left(p, s_{j}\right)$. Letting $\left(c_{x}, c_{y}\right)$ and $\left(d_{x}, d_{y}\right)$ be two endpoints of $s_{j}$, one of possible equations is

$$
\frac{\left|\left(a_{y}-b_{y}\right) x+\left(b_{x}-a_{x}\right) y+a_{x} b_{y}-a_{y} b_{x}\right|}{\left(x-a_{x}\right)^{2}+\left(y-a_{y}\right)^{2}}=\frac{\left|\left(c_{y}-d_{y}\right) x+\left(d_{x}-c_{x}\right) y+c_{x} d_{y}-c_{y} d_{x}\right|}{\left(x-c_{x}\right)^{2}+\left(y-c_{y}\right)^{2}},
$$

from which we have an equation for the corresponding Voronoi edge. If we partially differentiate the equations for $\mu\left(p, s_{i}\right)$ and $\mu\left(p, s_{j}\right)$ by $x$ and $y$ and putting them equal to 0 , then we have 4 different equations. Solving the system of equations, we have a constant number of points, which are candidates for peak positions. Finally, we evaluate the measures at those points and find a point among them that maximizes the measure. Fig. 10 illustrates terrains associated with polygons with optimal points highlighted.
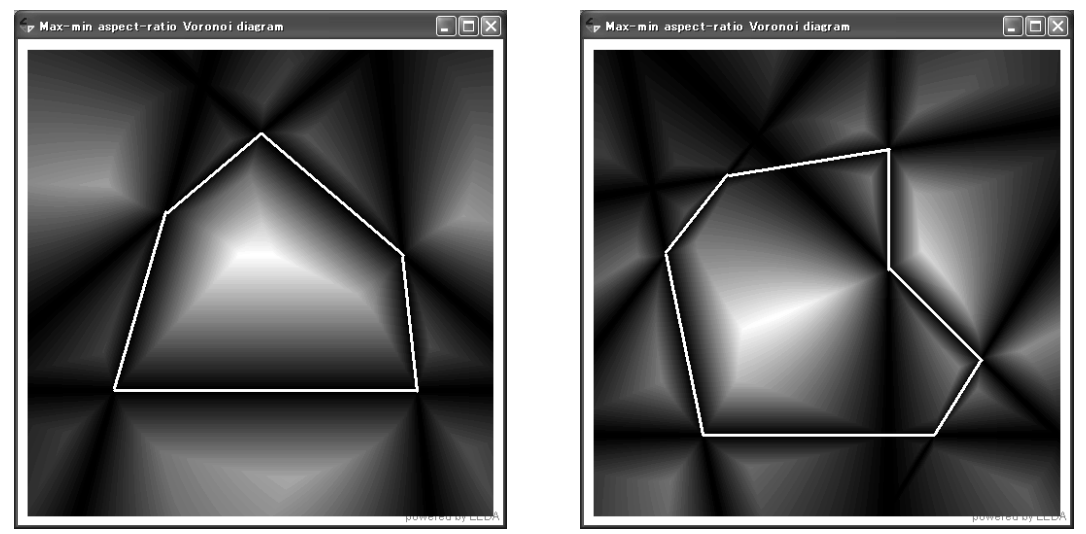

Fig. 10. Terrains on the minimum aspect-ratio Voronoi diagrams. Darker pixels have lower heights. The peak is highlighted.

\section{Concluding remarks}

In this paper we have presented a new family of Voronoi diagrams for a set of line segments or a polygon based on various measures on goodness of triangles. We have succeeded in characterizing their common combinatorial and structural properties. We have also presented a generic scheme for finding an optimal point with respect to a specified measure using the Voronoi diagram as guide. Unfortunately, our complexity result of $O\left(n^{2+\varepsilon}\right)$ is not encouraging for practical applications, but this is just an upper bound on the worst case complexity. Since the worst case is not known, especially for the cases where the set of line segments form a convex or starshaped polygon, it may be possible to lower the complexity for those cases. More experimental works are required to judge whether this idea is useful for practical use, which is left for future work. 
It is known in [9] that the problem of finding a point in a star-shaped polygon that maximizes the minimum visual angle when the point is connected to all the vertices of the polygon by straight edges is formulated as an LP-type problem and thus it can be solved by implementing $O(n)$ basic operations in the framework. So, it is more efficient than our approach based on the angular Voronoi diagram. Although it is hard to describe in limited space, there is an application in which we are required to find a point that maximizes the smallest visual angle in a starshaped polygon in some region bounded by some planar curves such as circular arcs. In such cases the diagram is useful and expected to be efficient because we do not need the whole diagram but just a part of it in the restricted area.

Acknowledgment. The part of this research was partially supported by the Ministry of Education, Science, Sports and Culture, Grant-in-Aid for Scientific Research on Priority Areas. Work of the first author was partially supported by the Ministry of Education, Science, Sports and Culture, Grant-in-Aid for Scientific Research (B). We also thank K. Ushikubo for his survey and programming. The first author would like to thank Franz Aurenhammer and his group for their discussions.

\section{References}

[ 1 ] P. Agarwal, O. Schwarzkopf and M. Sharir, The Overlay of Lower Envelopes and its Applications. Discrete \& Computational Geometry, 15 (1996), 1-13.

[ 2 ] T. Asano, Aspect-ratio Voronoi Diagram with Applications. Proc. International Symposium on Voronoi Diagram in Science and Engineering, Banff, Canada, 2006, 217-223.

[ 3 ] T. Asano, N. Katoh, H. Tamaki and T. Tokuyama, Angular Voronoi Diagram with Applications. Proc. International Symposium on Voronoi Diagram in Science and Engineering, Banff, Canada, 2006, 32-39.

[4] F. Aurenhammer, Voronoi Diagrams-A Survey of a Fundamental Geometric Data Structure. ACM Computing Surveys, 23 (1991), 345-405.

[ 5] H. Edelsbrunner and N. Shah, Incremental topological flipping works for regular triangulations. Proc. of the 8th ACM Symp. on Computational Geometry 1992, 1992, 43-52.

[6] L.A. Freitag and P. Plassmann, Local optimization-based simplicial mesh untangling and improvement. Int. J. for Numerical Methods in Engineering, 49 (2000), 109-125.

[ 7 ] D. Halperin and M. Sharir, Almost tight upper bounds for the single cell and zone problems in three dimensions. Proc. Symp. on Comput. Geom., 1994, 11-20.

[ 8 ] LEDA: A library for Efficient Data Types and Algorithms. Algorithmic Solutions Software GmbH, Germany.

[ 9 ] J. Matoušek, M. Sharir and E. Welzl, A Subexponential Bound for Linear Programming. Algorithmica, 16 (1996), 498-516.

[10] S.A. Mitchell and S.A. Vavasis, Quality Mesh Generation in Three Dimensions. Proc. of the Eighth Annual Symp. on Computational Geometry, 1992, 212-221.

[11] A. Okabe, B. Boots and K. Sugihara, Spatial Tessellations, Concepts and Applications of Voronoi Diagrams. John Wiley \& Sons, New York, 1992.

[12] J.R. Shewchuk, Delaunay refinement algorithms for triangular mesh generation. Comput. Geom., Theory and Appl., 22 (2002), 21-74.

[13] K. Shimada and D.C. Gossard, Bubble mesh: automated triangular meshing of non-manifold geometry by sphere packing. Proc. of the Third Symposium on Solid Modeling and Applications, 1995, 409-419. 